\title{
PROBLEM OF MATHEMATICAL DEDUCTION OF THE EXISTENCE OF BLACK HOLES
} YUAN-SHUN CHIN (YUANXUM QIN)

\author{
Institute of Applied Mathematics \\ Academia Sinica \\ Beijing, China \\ and \\ Department of Mathematics \\ University of Florida \\ Gainesville, Florida U.S.A.
}

(Recelved August 4, 1988 and in revised form December 13, 1988)

ABSTRACT. The mathematical proof of existence of Black Hole is based on the assumption of mass being independent of speed. Considering the effect of special relativity of the dependence of mass with speed there is no Black hole.

KEY WORDS AND PHRASES. Black holes and relativity. 1980 AMS SUBJECT CLASSIFICATION CODE. 83.

1. BLACK HOLE OF LAPLACE BASED ON NEWTONIAN THEORY.

In 1792 Laplace suggested that there might be "some very dense and very hot star, that cannot be seen." His reasoning is as follows:

Suppose there is a star with mass $M$ and radius $r$ which is so dense such that its gravitational force produces the effect that the light particles emitted from its surface will eventually be attracted back to $1 t$, hence it cannot be seen at far distance, although it might be very hot because of its extraordinary large density.

This is the reasoning of existence of Laplace-Newton Black Hole.

2. MATHEMATICAL DEDUCTION OF THE CRITICAL RADIUS OF LAPLACE-NEWTON BLACK HOLE.

Suppose a star with mass $M$ is situated at the origin of the coordinate system, 1.e. $R=0$. A particle with mass $m$ falls from $R=\infty$ by the gravitational force of Newton toward the star $M$, with the initial velocity $v=0$. The equation of motion $1 \mathrm{~s}$ then

$$
\frac{\mathrm{d}}{\mathrm{dt}}\left(\mathrm{m} \frac{\mathrm{dR}}{\mathrm{dt}}\right)=-\frac{\mathrm{GMm}}{\mathrm{R}^{2}}
$$

In Newtonian mechanics, mass does not change with speed,

$$
\mathrm{m}=\text { const. }
$$

so $m$ can be cancelled out to get

$$
\frac{d^{2} R}{d t^{2}}=-\frac{G M}{R^{2}} .
$$

The integration of (3) with the initial conditions:

$$
t=0, R=\infty, \frac{d R}{d t}=0,
$$

gives the result: 


$$
\frac{1}{2}\left(\frac{\mathrm{dR}}{\mathrm{dt}}\right)^{2}=\frac{\mathrm{GM}}{\mathrm{R}}
$$

or

$$
v^{2}=\frac{2 G M}{R}
$$

and

$$
\nu=\sqrt{\frac{2 G M}{R}}
$$

Now, if the radius $r$ of the star is so small such that

$$
\sqrt{\frac{2 G M}{r}}>c \text {, (velocity of 1ight) }
$$

then the light particles (considered as material mass points in Newtonian sence) emitted from the surface of the star cannot reach point at infinity and will be attracted back eventually to the star again.

The critical radius $R_{L N}$ of a star with mass $M$ then satisfies

$$
\sqrt{\frac{2 G M}{R_{L N}}}=c
$$

or

$$
\mathrm{R}_{\mathrm{LN}}=\frac{2 \mathrm{GM}}{\mathrm{c}^{2}} \text {. }
$$

So far all we have done is within Newtonian physics.

3. SPECIAL RELATIVITY DENIES THE EXISTENCE OF LAPLACE-NEWTON BLACK HOLE.

In the two centuries that have past, no Laplace-Newton black holes have been detected. Theoretically the reasoning for existence of Laplace-Newton black hole can be discarded as follows:

In special relativity mass is not a constant as Newton assumed in (2.2), but changes with speed in the following way:

$$
m=\frac{m_{0}}{\sqrt{1-\left(\frac{v}{c}\right)^{2}}}
$$

so that $m$ cannot be cancelled out in (2.1), and one gets the equation:

with initial values $(2.4)$.

$$
\frac{d}{d t}\left(\frac{d R}{d t} \frac{m_{0}}{\sqrt{1-\left(\frac{d R}{c d t}\right)^{2}}}\right)=-\frac{G M}{R^{2}} \frac{m o}{\sqrt{2-\left(\frac{d R}{c d t}\right)^{2}}}
$$

Now $m_{0}$ does not change with speed so it can be cancelled from (3.2), and integration of (3.2) with condition (2.4) gives

So if one puts

$$
v^{2}=\left(\frac{v}{c}\right)^{2}=\left(\frac{d R}{c d t}\right)^{2}=1-\exp \left(\frac{-2 G M}{c^{2} R}\right)
$$

$$
\mathrm{v}=\mathrm{c}, \text { i.e. } \mathrm{V}=1 \text {, }
$$

then

$$
\mathbf{R}=0 \text {. }
$$


Hence, based on special relativity, the Laplace-Newton Black Hole does not exist.

The crucial point is that the existence of critical radius

$$
\mathrm{R}_{\mathrm{LN}}=\frac{2 \mathrm{GM}}{2}
$$

of the Laplace-Newton black hole $1 \stackrel{c}{s}$ deduced mathematical without considering the effect of mass changing with speed.

This is quite understandable, because special relativity appears long after Laplace.

4. THE PROBLEM OF MATHEMATICAL DEDUCTION OF CRITICAL RADIUS R OS OF OPPENHEIMERSCHAWARZCHILD BLACK HOLE.

In 1939 Oppenheimer deduced the theoretical existence of a black hole based on Schwarzschild Line Element:

$$
\mathrm{ds}^{2}=\left(1-\frac{2 \mathrm{GM}}{\mathrm{Rc}}\right) \mathrm{dT}^{2}-\left[\frac{\mathrm{dR} \mathrm{R}^{2}}{1-\frac{2 \mathrm{GM}}{\mathrm{Rc}^{2}}}+(\mathrm{R} \sin \theta)^{2} \mathrm{~d} \phi^{2}+\mathrm{R}^{2} \mathrm{~d} \theta^{2}\right] .
$$

From (4.1) one can see at once that if the factor

$$
1-\frac{2 G M}{R c^{2}}=0 \text {, }
$$

then the Schawarzschild Singularity appears at

i.e.

$$
\begin{aligned}
& 1-\frac{2 M G}{\operatorname{Ros} c^{2}}=0 \\
& R_{o s}=\frac{2 G M}{c^{2}} .
\end{aligned}
$$

Any star with mass $M$ and with radius smaller than $R_{\text {os }}$ is an Oppenheimer-Schwarzschild Black Hole.

In the standard textbook of relativity by P.G.Bergman [1] with forward by $A$. Einstein there is a remark:

"In nature, mass is never sufficiently concentrated to permit a Schwarzschild Singularity to occur in empty space."

This remark has not obtained sufficient attention from scientists working on black holes. Many experiments are conducted to find Oppenheimer-Schwarzschild black holes in the universe, but few people considered the assumptions leading to the theoretical prediction of the existence of Oppenheimer-Schwarzschild black holes.

Although the theory of relativity is fundamentally different from the theory of space-time of Newton-Galileo, a crucial question is why the critical radius $R_{L N}$ of Laplace-Newton black hole is identically equal to the critical radius $R_{0 S}$ of Oppenheimer-Schwarzschild black hole?

$$
\mathrm{R}_{\mathrm{LN}} \equiv \mathrm{R}_{\mathrm{OS}} ?
$$


Is this a relation by chance or a certainty by same assumption? The answer is that they are the same mathematical deductions based on the same assumption that mass does not change with speed.

Let us look at how the constant of Gravitation $G$ gets into the theory of General Relativity.

In mathematical deduction of Schwarzschild line element (4.1) one arrives at the metric tensor with a component:

$$
g_{44}=e^{B}\left(1-\frac{\alpha}{R}\right)
$$

with two integration constants $\alpha$ and $\beta$ to be determined.

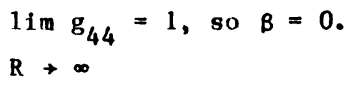

To determine the integration constant $\alpha$, the Newtonian potential of a point of mass $M$ which creates the filed with (4.4) is

$$
G_{M}=-\frac{\alpha}{2 R}
$$

On the other hand, the Newtonian potential of a point with mass $M$ is given by

$$
G_{M}=-\frac{G M}{R}
$$

Hence one gets the integration constant $\alpha$ as

$$
\alpha=2 \mathrm{GM}
$$

It is clear that the gravitational constant $G$ gets into the theory of general relativity through the Newtonian potential $G_{M}$ of (4.5), a result of (2.5) based on the assumption (2.2).

Mass does not change with speed.

Hence the identity (4.3) becomes reasonably expectable, because the two critical radius $R_{L N}$ and $R_{O S}$ are deduced mathematically on the same assumption (2.2).

Since special relatively replaces (2.2) by (3.1) to discard the existence of the Laplace-Newton black hole, it is naturally to expect that replacing (2.2) by (3.1) will also solve the difference of the mathematical deduction of existence of the Oppenheimer-Schwarzschild black hole based on the assumption of (2.2) and the reality that in nature one has not found any real Oppenheimer-Schwarzschild black hole, because mass does change with speed according to (3.1). 
5. MATHEMATICAL DEDUCTION OF SCHWARZSCHILD LINE ELEMENT FROM SPECIAL RELATIVITY AND NEWTONIAN MECHANICS.

We use three simple steps to deduce it.

(i) Let a heavy mass $M$ be situated at the origin $(0,0,0)$. From Special Relativity one has:

$$
d s^{2}=d T_{L}^{2}-\left(d x_{L}^{2}+d y_{L}^{2}+d z_{L}^{2}\right)
$$

for the local time-space coordinates $\left(x_{L}, y_{L}, z_{L}\right)$, or in polar coordinates $\left(R_{L}, \theta, \phi\right):$

$$
d s^{2}=d T_{L}^{2}-\left(d R_{L}^{2}+\left(R_{L} \sin \theta\right)^{2} d \phi+R_{L}^{2} d \theta^{2}\right)
$$

(11) Let an Einstein elevator falling from point at infinity with zero initial speed toward this mass $M$ under the Newtonian gravitation law. Then the speed is:

$$
v=\sqrt{\frac{2 G M}{R_{\infty}}}
$$

Here $R_{\infty}$ means measured by a rod at infinity, 1.e. no contraction in Newtonian mechanics, or

$$
V=\frac{v}{c}=\sqrt{\frac{2 G M}{R_{\infty} c}} .
$$

(iii)From the Einstein elevator, by special relativity one has

and

$$
\mathrm{dT}_{\mathrm{L}}=\sqrt{1-\mathrm{v}^{2} \mathrm{dT}_{\infty}}
$$

$$
\mathrm{dR}_{L}=\frac{\mathrm{dR}_{\infty}}{\sqrt{1-\mathrm{v}^{2}}} .
$$

These are standard realtions of special relativity for local time-space.

There is no relative velocity in $(\phi, \theta)$ directions, so there is no Lorentz factor for $(\phi, \theta)$. Putting (5.3), (5.4) and (5.5) into (5.2), one gets at once the Schwarzschild line element:

$$
d s^{2}=\left(1-\frac{2 G M}{c^{2} R_{\infty}}\right) d T_{\infty}^{2}-\left[\frac{d R_{\infty}^{2}}{1-\frac{2 G M}{c^{2} R_{\infty}}}+\left(R_{\infty} \sin \theta\right)^{2} d \phi^{2}+R_{\infty}^{2} d \theta\right] .
$$

From this type of deduction we can see that the relative velocity $V$ comes from Newtonian mechanics.

Now if we replace $(2.3)$ by $(3.1)$ as we have done In section III, we shall have

and

$$
v^{2}=\left(\frac{v}{c}\right)^{2}=1-\exp \left(-\frac{2 G M}{R_{\infty} c^{2}}\right)
$$

so that the line element becomes:

$$
1-v^{2}=\exp \left(-\frac{2 G M}{R_{\infty} c^{2}}\right)
$$




$$
d s^{2}=\left(\exp \left(-\frac{2 G M}{R_{\infty} c^{2}}\right)\right) d T_{\infty}^{2}-\left[\left(\exp \left(\frac{2 G M}{R_{\infty} c^{2}}\right)\right) d R_{\infty}^{2}+\left(R_{\infty} \sin \theta\right)^{2} d \phi^{2}+R_{\infty}^{2} d \theta^{2}\right] .
$$

Hence there is no singularity in the line element (5.7) except the origin, which will be discussed in the last section.

Again we get line element without a black hole by considering the effect that mass does change with speed.

6. THE EXPERIMENTAL TESTS OF LINE ELEMENT (5.7).

$$
\exp \left(-\frac{2 G M}{R c^{2}}\right)=1-\frac{2 G M}{R c^{2}}+\cdots
$$

So (5.7) is the same as Schwarzschild line element in first approximation, the gravitational red shift and the deflection of 11 ght are just the same. The crucial test is the precession of the periherion of Mercury, which is an effect of second order approximation.

The discrepancy is

$$
\Delta=\frac{24 \pi^{3} a^{2}}{\left(1-e^{2}\right)(c r)^{2}} \text { (Angle in radiants per period) }
$$

The line element (5.7) gives $\frac{2}{3}$ of this value. The problem is now: Where is the $\frac{1}{3}$ of this value?

The answer comes from the spectal relativity again. From the Lorentz transformation:

$$
\mathrm{X} *=\frac{\mathrm{X}-\mathrm{VT}}{\sqrt{1-\mathrm{v}^{2}}} \quad \mathrm{~T} *=\frac{\mathrm{T}-\mathrm{VX}}{\sqrt{1-\mathrm{v}^{2}}}
$$

so in one turn of the oribit there is a time delay of the magnitude:

$$
\frac{V X}{T}=\frac{\frac{2 \pi a}{c \tau} 2 \pi a}{c \tau}=\left(\frac{2 \pi a}{c \tau}\right)^{2} \text {, }
$$

for one period $\tau$. Change this angle into a radian system, 1.e. mulitiply it by $2 \pi$, to obtain:

$$
2 \pi \frac{V X}{T}=\frac{8 \pi^{3} a^{2}}{(c \tau)^{2}}=\frac{1}{3} \Delta
$$

This discrepancy is then closed, 1.e. two omissions cancelled each other just to get the result compatible with the experimental results. Both these two omissions are required by the special relativity.

Like the Laplace-Newton black hole the Oppenheimer-Schwarzschild black hole is 
also a mathematical deduction based on Newtonian mechanics of mass indpendent of speed, and is incompatible with spectal relativity. Hence this problem is solved mathematically, because it is a mathematical deduction.

7. INTERIOR LINE ELEMENT.

(5.7) is the exterior line element, good for space outside of the star. Inside the star we should use the interlor line element.

We use the Newtonian relation:

$$
M(R)=\frac{4 \pi}{3} \rho R^{3}
$$

with the boundary condition:

$$
\mathrm{V}_{\mathrm{R}=\mathrm{r}}^{2}=1-\exp \left(\frac{-2 \mathrm{GM}}{\mathrm{c}^{2}}\right)
$$

where $r$ is the radius of the star.

Integration gives the relation:

$$
1-v^{2}(R)=\exp \left(\frac{2 \pi}{3 c^{2}} \rho G\left(R^{2}-3 r^{2}\right)\right) \quad \text { for } 0<R<r
$$

The interior line element is then

$$
d s^{2}=\left(1-v^{2}(R)\right) d T^{2}-\left[\left(\frac{d R^{2}}{1-v^{2}(R)}\right)+(R \ln \theta)^{2} d \phi^{2}+R^{2} d \theta^{2}\right.
$$

Now for $R=0$,

$$
-v^{2}(0)=\exp \left(\frac{-2 \pi \rho G r^{2}}{c^{2}}\right)
$$

Hence there is no singular point at the origin. This interior line element is due to Professor H.W. Peng, the Honorary Director of Institute of Theoretical Physics, Academia Sinica, Beijing, PRC.

The problem of mathematical deduction of the existence of blackholes based on the assumption that mass does not change with speed is then completely solved.

The results in this paper have been published partially in [2] in Chinese.

\section{REFERENCES}

1. BERGMAN, P.G., Introduction to the Theory of Relativity (with forward by A. Einstein), 1948 Prentice-Ha11, Inc.

2. CHIN, Y.S., Theory of Local Instantaneous Symmetry of Space and Time under variable velocity conditon. Acta. Physica. Sinica 25 (1976), 355-361. 


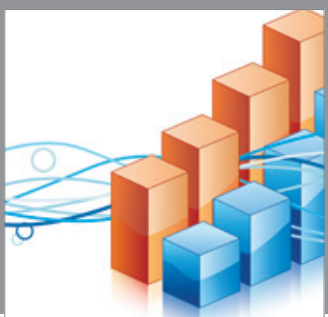

Advances in

Operations Research

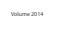

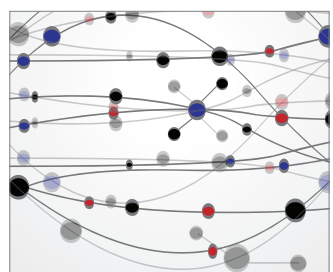

\section{The Scientific} World Journal
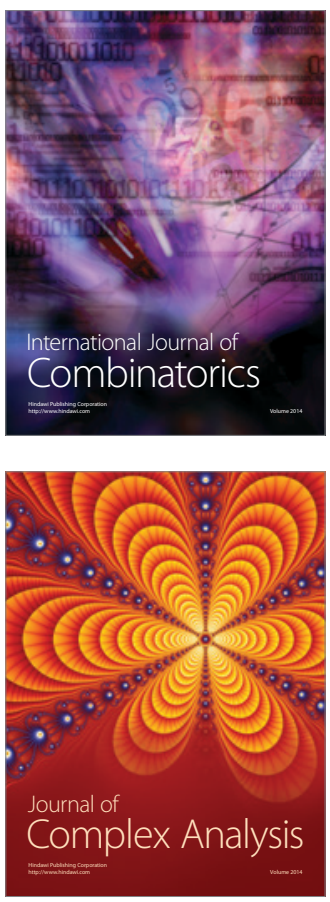

International Journal of

Mathematics and

Mathematical

Sciences
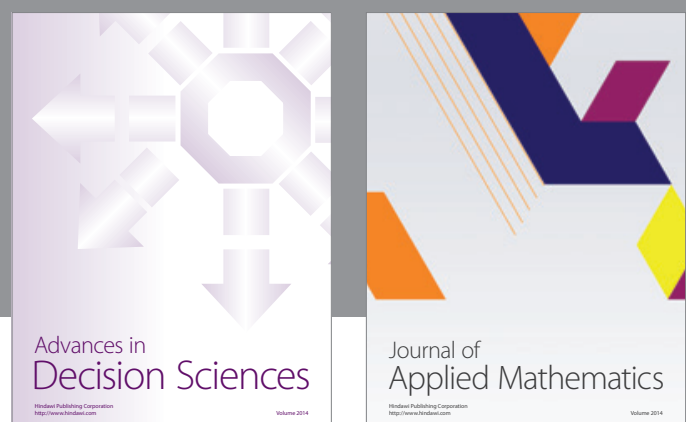

Journal of

Applied Mathematics
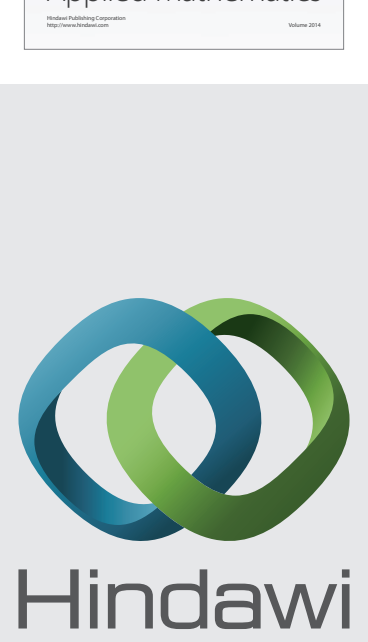

Submit your manuscripts at http://www.hindawi.com
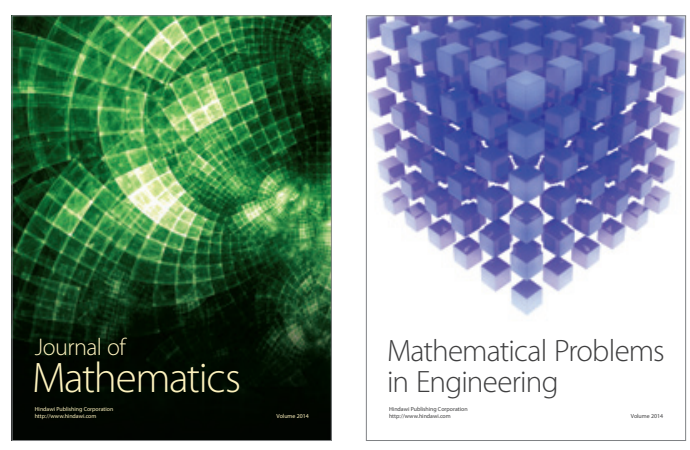

Mathematical Problems in Engineering
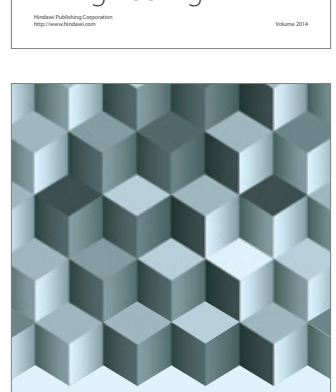

Journal of

Function Spaces
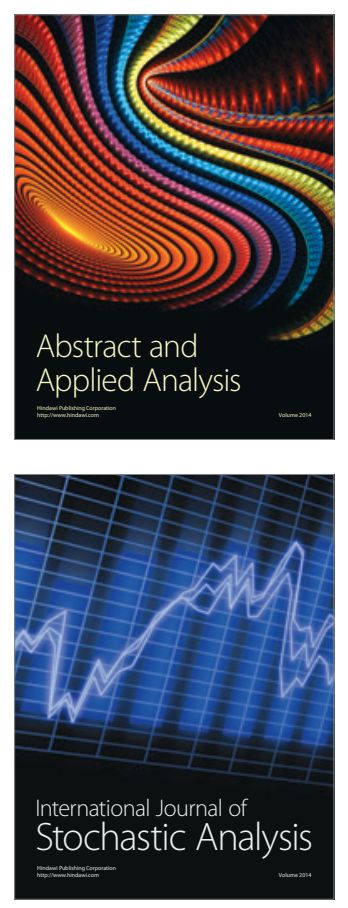

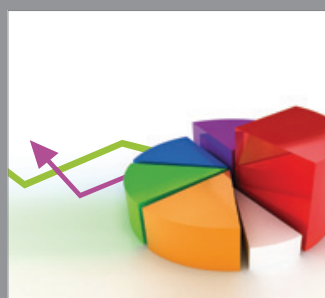

ournal of

Probability and Statistics

Promensencen
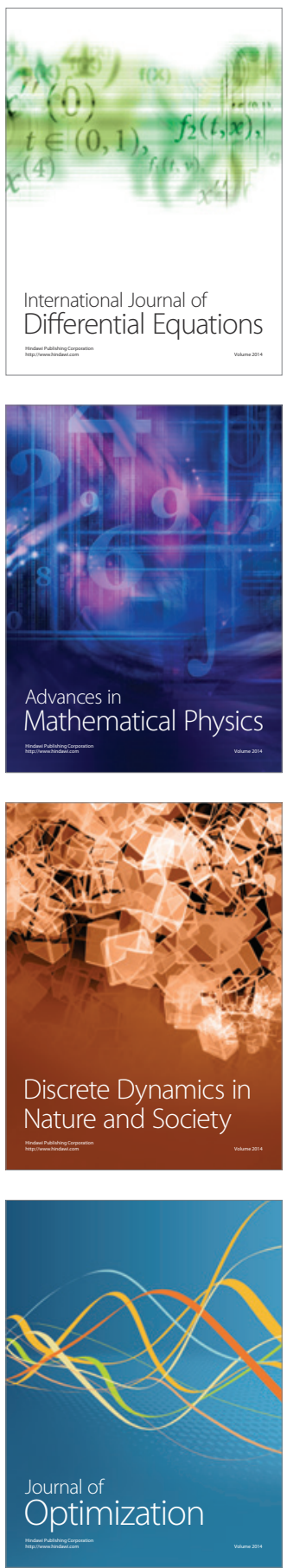